\title{
非热电子特性的微波诊断*
}

周爱华 (1) (2) 张 捷 (2) 张和 ${ }^{\left({ }^{+}\right.}{ }^{(2)}$

(1) 中国科学院射电天文联合实验室, 南京 210008; (2) 中国科学院紫金山天文台, 南京 210008)

\section{关键词太阳微波爆 非热电子}

太阳微波爆, 一般是非热电子与磁场相互作用的回旋同步加速辐射产生的, 因而微波爆观 测应具有诊断非热电子和磁场信息的能力. 但由于这种辐射在计算上的复杂性很难将这些信息 确切地提取出来, 直到 1982 年 Dulk 和 Marsh ${ }^{[1]}$ 给出这种辐射的近似式后, 人们才开始利用 其中的谱翻转频率 $v_{p}$ 的近似式, 结合其它资料或假设将方程中的两个未知数 (磁场强度 $B$ 和非热电子柱密度 $N L)$ 中的一个视为已知, 再求第二个 ${ }^{[2 \sim 4]}$, 这样所得的 $B$ 和 $N L$ 的解是不 定的. 最近我们提出一种新方法, 仅仅根据微波爆本身的观测, 在均匀源的假设下, 第一次获 得了磁场 $B$ 和柱密度 $N L$ 的独立解及误差估计 ${ }^{55}$. 6. 本文利用该方法进一步导得非热电子的 能量分布 $n(E)$ 和它们所携带的能流 $\varepsilon_{\mathrm{F}}$ 的表达式, 使我们能从微波爆发资料第一次获得非热 电子特性的所有信息, 无疑这对耀斑能量释放研究、耀斑的热与非热形成机理的研究提供了 定量依据.

在一个均匀的和光薄 $\left(\tau_{v} \ll 1\right)$ 的微波源中亮度温度 $T_{\mathrm{bv}}$ 可简化为

$$
T_{\mathrm{bv}} \approx T_{\mathrm{eff}} \tau=\frac{c^{2}}{k v^{2}} \eta_{v} L,
$$

其中 $\eta_{v}$ 为非热电子发射系数, $L$ 为源大小, 其它为常用物理量. 由 (1) 式可导得非热电子柱 密度 $N\left(>E_{0}\right) L$ 的表达式

$$
N L=T_{\mathrm{b} v}\left(\frac{k v^{2}}{c^{2}}\right)\left(\frac{\eta_{v}}{N B}\right)^{-1} B^{-1} .
$$

当 $2 \leqslant \delta \leqslant 7$ 时, 在回旋同步加速辐射情况下发射系数和经验公式为 ${ }^{[1]}$

$$
\frac{\eta_{v}}{B N} \approx 3.3 \times 10^{-24-0.52 \delta}(\sin \theta)^{-0.43+0.658}\left(\frac{v}{v_{B}}\right)^{1.22-0.90 \delta} .
$$

由文献[5] 给出磁场 $B$ 的计算公式为

$$
B \approx\left(\frac{c^{2}}{k T_{\mathrm{bo}} A_{1}} v_{p}^{1.30+0.988 \delta} v^{-0.78-0.90 \delta}\left(2.8 \times 10^{6}\right)^{-2.52-0.08 \delta}\right) \frac{1}{0.52+0.08 \delta},
$$

其中 $A_{1}=4.24 \times 10^{14+0.305}(\sin \theta)^{0.34+0.07 \delta}$. (4) 式中的 $v_{p}$ 和 $v_{B}$ 分别为爆发谱翻转频率和电子回旋频 率, $\theta$ 为 $\boldsymbol{k}$ 与 $\boldsymbol{B}$ 的夹角, $\delta$ 为非热电子能谱指数, 它由射电谱光薄处谱指数 $\alpha$

$$
S_{v} \propto v^{\alpha}
$$

1995-03-20 收稿, 1995-08-01 收修改稿

*国家自然科学基金和中国科学院天文委员会资助项目 
决定, 根据 (3) 式, $\delta$ 与 $\alpha$ 的关系为

$$
\delta=(1.22-\alpha) / 0.9 .
$$

(4) 式表明, 在射电爆发谱参数 $v_{p}, \alpha$ 和 $T_{\mathrm{bv}}$ 已知情况下, 磁场强度 $B$ 可求. 一旦 $B$ 求得, 则非 热电子柱密度 $N L$ 由 (2) 式也即可求. 在已知源大小 $L$ 的情况下, $N\left(>E_{0}\right)$ 值可求, 然后非热 电子的能量分布

$$
n(E)=G E^{-\delta}
$$

也可决定，其中

$$
G=N(\delta-1) E_{0}^{(\delta-1)},
$$

这时非热电子所携带的能流 $\varepsilon_{\mathrm{F}}\left(10^{7} \mathrm{~J} \cdot \mathrm{cm}^{-2} \cdot \mathrm{s}^{-1}\right)$ 亦可计算,

$$
\varepsilon_{\mathrm{F}}=\int_{E_{0}}^{E_{m}} E v n(E) \mathrm{d} E,
$$

$E_{0}, E_{m}$ 分别为非热电子的低能和高能截止值. 将(7)式代人 (9) 式, 可得

$$
\begin{gathered}
\varepsilon_{\mathrm{F}}=\frac{1.76 G}{(2.5-\delta)}\left(E_{m}^{(2.5-\delta)}-E_{0}^{(2.5-\delta)} \quad(2.5<\delta<2.5),\right. \\
\varepsilon_{\mathrm{F}}=1.76 G \ln E_{m} \quad(\delta=2.5) .
\end{gathered}
$$

我们取 $\theta=45^{\circ}, L=9.4 \times 10^{7} \mathrm{~cm}$, 由 (10) 式计算了 $\varepsilon_{\mathrm{F}}$ 值随非热电子能谱指数 $\delta$ 和谱翻转 频率 $v_{p}$ 的分布 (见图 1(a), (b)). 图中 3 条曲线分别对应亮度温度 $T_{\mathrm{b}}\left(v=3 \times 10^{10} \mathrm{~Hz}\right.$ ) 为 $10^{9} \mathrm{~K}, 10^{8} \mathrm{~K}$ 和 $10^{7} \mathrm{~K}$ 的情况. 计算表明, 当射电观测谱愈软 ( $\alpha$ 愈大)、谱翻转频率 $v_{p}$ 愈低和亮度温度 $T_{\mathrm{b} v}$ 愈高时, 所相应的非热电子流携带的能流 $\varepsilon_{\mathrm{F}}$ 值愈高.

对 $\varepsilon_{\mathrm{F}}$ 的误差作了估计, 在取 $v_{p}$ 、射电流量密度 $S_{v}$ 和亮度温度 $T_{\mathrm{b}}$ 的误差分别在 \pm 0.10 、
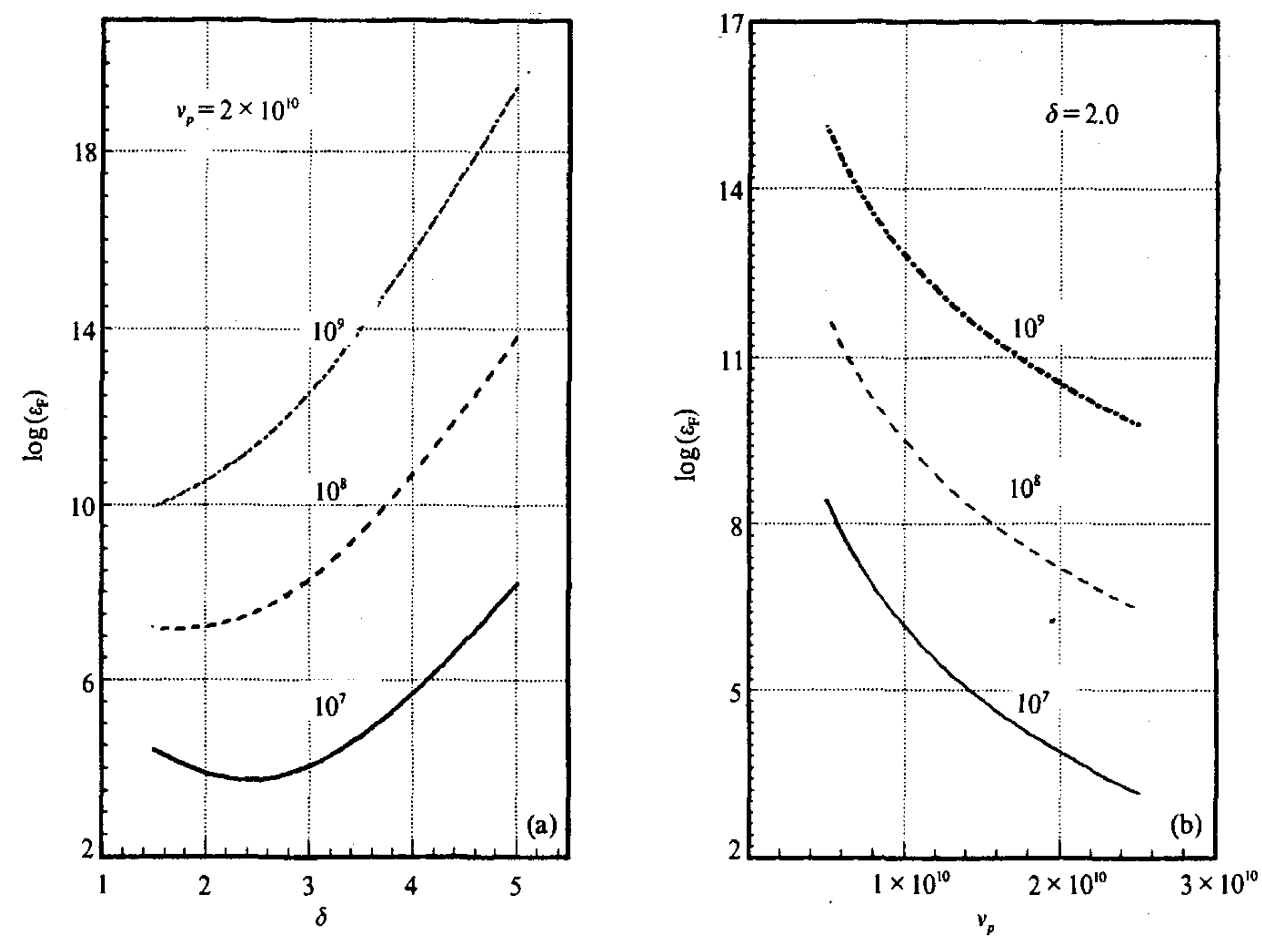

图 1 非热电子携带的能流 $\varepsilon_{\mathrm{F}}\left(10^{7} \mathrm{~J} \cdot \mathrm{cm}^{-2} \cdot \mathrm{s}^{-1}\right)$ 与其能谱指数 $\delta(\mathrm{a})$ ）谱翻转频率 $v_{p}(\mathrm{~b})$ 的关系 其中亮度温度 $T_{\mathrm{b}}\left(v=3 \times 10^{10} \mathrm{~Hz}\right)$ 分别为: $10^{9}(\mathrm{~K})(-\cdots \cdot-), 10^{8}(\mathrm{~K})(-\cdots-)$ 和 $10^{7}(\mathrm{~K})(-$ ) 
\pm 0.05 和 \pm 0.30 的情况下, 结合文献[1] 的近似式 $\frac{\eta_{v}}{B N}$ 的误差考虑 $( \pm 0.26)$, 求得 $\varepsilon_{\mathrm{F}}$ 的相对 误差为 \pm 0.80 . 由于本工作受文献 [1] 的回旋同步加速理论限制, 本文结果仅适用 $2 \leqslant \delta \leqslant 7$, $10 \leqslant v / v_{B} \leqslant 100$ 和 $\theta$ 不为极端角情况.

本文表明, 只需由微波爆发谱本身提供的一套谱参数, 就可以获得非热电子的柱密度 $N L$ 、能谱分布 $n(E)$ 和能流 $\varepsilon_{\mathrm{F}}$, 其他波段的资料和除均匀源之外的过多假设不再需要. 非热电 子这些特征量的定量计算, 使有可能深人研究耀斑的能量释放过程和这些非热电子流所带来 的对耀斑光学波段的影响.

致谢与徐之材研究员对误差方面的有益讨论及王欣东在计算等方面的协助, 作者表 示感谢。

\section{参考文献}

1 Dulk G A, Marsh K A. Simplified expressions for the gyrosynchrotron radiation from mildlyrelativistic nonthermal and thermal electrons. Astrophys J, 1982, 259:350

2 Gary D. The numbers of fast electrons in solar flares as deduced from hard X-ray and microwave spectral data. Astrophys J, 1985, 297: 799

3 周爱华. 从一个微波 C 型爆推算到的高能电子数。天体物理学报, 1992, 12(2): 148

4 Wang H, Gary D E, Lim J. Microwave spectral imaging and hard X-ray observations of a solar limb flare. Astrophys J, 1994, 433: 379

5 Zhou Ai-hua, Karlicky M. Magnetic field estimation in synchrotron radiation sources. Solar Phys, 1994, 153:441

6 Zhou A H. Magnetic field strength estimation in microwave radio sources. Astrophysics and Space Scienc, 1994, 222: 107 